\title{
Paraurethral cyst in a newborn
}

\author{
Raziye Ergun ${ }^{1}$, Duran Yildiz ${ }^{2}$, Cagri Akin Sekerci ${ }^{3}$, Hasan Kahveci ${ }^{2}$ \\ ${ }^{1}$ Erzurum Regional Training and Research Hospital, Pediatric Urology, Erzurum, Turkey; \\ 2 Erzurum Regional Training and Research Hospital, Neonatology, Erzurum, Turkey; \\ ${ }^{3}$ Marmara University Pendik Training and Research Hospital, Pediatric Urology, Istanbul, Turkey.
}

\begin{abstract}
Summary Paraurethral cysts are rare and occur with obstruction of the Skene duct. In this case, we aimed to present a paraurethral cyst in a baby girl. A 4-day-old newborn was consulted for pediatric urology because of an interlabial mass. In genital examination, a noninvasive mass measuring $2 \times 1.5 \mathrm{~cm}$ was observed in the interlabial region, and the introitus was completely closed. Ultrasonography showed a cystic lesion localized on the right side of the urethra with a smoothly contoured, thin-walled, hypoechoic structure. The patient underwent puncture under sterile conditions. The content of the mass was mucoid and clear fluid. Paraurethral cysts are often asymptomatic and benign. There is no definitive consensus on treatment.
\end{abstract}

KEY WORDS: Newborn; Paraurethral cyst.

Submitted 26 July 2019; Accepted 31 January 2020

\section{INTRODUCTION}

Paraurethral cysts are a rare cause of intralabial mass in neonatal period. They develop due to congenital obstruction of the ducts of the paraurethral glands.

The incidence of paraurethral cysts has been reported as 1.038 to 7.246 live births (1). Paraurethral cysts are usually asymptomatic, 6-10 $\mathrm{mm}$ in size, cystic and soft masses in the physical examination. Most of them spontaneously resolve. In this article, we aimed to present a case of paraurethral cyst which was treated by needle aspiration in early neonatal period.

\section{Case report}

On the fourth day after birth, a baby girl was admitted to the neonatal intensive care unit with cyanosis. She was born from non-consanguineous parents, following an uneventful second pregnancy for her 40-year-old mother, by the normal spontaneous vaginal route at a private center. She was born at 37 weeks of gestation, with a weight of 2.200 grams $\left(3^{\text {rd }}-10^{\text {th }}\right.$ percentile) a height of $45 \mathrm{~cm}\left(25-50^{\text {th }}\right.$ percentile). In the systemic examination, the patient's general status was good.

The neonatal reflexes were active and alive, and her vital signs were normal. Moreover, the results of her respiratory, cardiovascular, neurological, and abdominal examinations were normal. In genital examination, a noninvasive mass measuring $2 \times 1.5 \mathrm{~cm}$ was observed in the interlabial region, and the introitus was completely closed. The patient had spontaneous urine output.
Complete blood count (CBC), C-reactive protein, and other biochemical tests were normal. Ultrasonography (US) showed a cystic lesion localized on the right side of the urethra with a smoothly contoured, thin-walled, hypoechoic structure that was unrelated to the surrounding tissues. Abdominopelvic US was normal.

The patient's oxygen saturation values returned to normal after cleaning nasal secretions with saline. Following the evaluation of pediatric urology, the mass was thought to be a paraurethral cyst. After written informed consent was obtained from the family, the patient underwent puncture under sterile conditions. The content of the mass was mucoid and clear fluid. After the procedure, the urethra and vagina were normal. It was observed that the cyst did not recur during follow up at the neonatal unit or after the patient's discharge.

\section{Discussion}

Paraurethral glands were first described by Alexander Johnston Chalmers Skene in 1880 (2). They are located in the distal part of the urethra and considered to be homologues of the prostate in females; they generate mucoid secretion with sexual stimulation (3). The distal urethra of an adult woman has between 6 and 30 ducts in the paraurethral glands, the largest of which is called the Skene duct. Paraurethral cysts are rare and occur with obstruction of the Skene duct. Although the etiology is not clear, it is thought that dislocation of the epithelium and inflammation may cause obstruction (4).

The diagnosis of paraurethral cyst in newborns is based on the localization of the cyst and relationship with the urethra. In paraurethral cysts, the urethra is free, and the urethral meatus can displace with the mass effect. Perineal ultrasound shows a smooth contoured simple cyst filled with mucoid fluid. On pathological examination, the internal surface of the cyst wall comprises transitional or squamous epithelium. Differential diagnoses of paraurethral cysts in newborns include imperforate hymen, Gardner canal cyst, Muller canal cyst, urethral prolapse, vaginal rhabdomyosarcoma, ectopic ureterocele, condyloma, urethral polyp, congenital lipoma, and vaginal prolapse. If paraurethral cysts are accompanied by lower urinary tract obstruction, further urological evaluation should also be performed.

There is no consensus about the treatment for paraurethral cysts because they are benign and often asympto- 
Figure 1.

View of the paraurethral cyst and aspirated fluid with the naked eye.

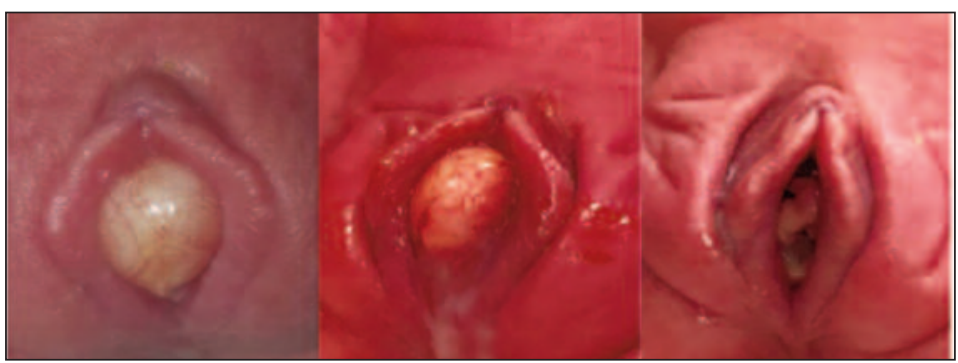

A

B

Fujimoto et al. reported that the five neonates with paraurethral cysts who were followed conservatively showed spontaneous recovery after 150 days on average (5). The most common method for surgical procedures is needle aspiration, which can often be applied at the bedside with a local anesthetic cream without the need for general anesthesia.

\section{REFERENCES}

1.Phupong V, Aribarg A. Management of Skene's duct cysts in newborn girls. BJU Int. 2000; 86:562.

2. Skene AJ. The anatomy and pathology of two important glands of the female urethra. The American Journal of Obstetrics and Diseases of Women and Children (18691919). 1880; 13:265.

3. Costantino E, Ganesan GS. Paraurethral cysts in newborn girls. BMJ case reports. 2016; 2016:bcr2016216689.

matic. Cyst excision, marsupialization, and needle aspiration, as in our case, are among the surgical methods that can be used in the treatment. Because of spontaneous resolution in neonates, a conservative approach is another alternative to surgery.

1B: Content of the aspirated cyst during operation.

1C: Image of vulva after operation (fifth day).
4. Merlob P, Bahari C, Liban E, Reisner S. Cysts of the female external genitalia in the newborn infant. Am J Obstet Gynecol. 1978; 132:607-10

5. Fujimoto T, Suwa T, Ishii N, Kabe K. Paraurethral cyst in female newborn: is surgery always advocated? J Pediatr Surg. 2007; 42:400-3.

\section{Correspondence}

Raziye Ergun, MD

raziye_ergun@hotmail.com

Erzurum Regional Training and Research Hospital, Pediatric Urology,

Erzurum (Turkey)

Duran Yildiz, MD

doktorduranyildiz@hotmail.com

Erzurum Regional Training and Research Hospital, Neonatology,

Erzurum (Turkey)

Cagri Akin Sekerci, FEBU, Assoc. Prof. (Corresponding Author)

cagri_sekerci@hotmail.com

Marmara University Pendik Training and Research Hospital,

Pediatric Urology, Istanbul (Turkey)

Hasan Kahveci, Assoc. Prof.

drhasankahveci@hotmail.com

Erzurum Regional Training and Research Hospital, Neonatology,

Erzurum (Turkey) 